\title{
Golf Swing Rotational Velocity: The Essential Follow-Through
}

\author{
Katherine M. Steele, $\mathrm{PhD}^{1,2,3}$, Eugene Y. Roh, $\mathrm{MD}^{1}$, Gordhan Mahtani, MS ${ }^{1,2}$, David W. Meister, $\mathrm{MD}^{4}$, \\ Amy L. Ladd, $\mathrm{MD}^{1}$, Jessica Rose, $\mathrm{PhD}^{1,2}$
}

${ }^{1}$ Department of Orthopaedic Surgery, Stanford University School of Medicine, Stanford, CA; ${ }^{2}$ Motion \& Gait Analysis Laboratory, Lucile Packard Children's Hospital, Stanford, CA; ${ }^{3}$ Department of Mechanical Engineering, University of Washington, Seattle, WA; ${ }^{4}$ Department of Orthopaedic Surgery, Medical College of Wisconsin, Milwaukee, WI, USA

Objective To evaluate if shoulder and pelvic angular velocities differ at impact or peak magnitude between professional and amateur golfers. Golf swing rotational biomechanics are a key determinant of power generation, driving distance, and injury prevention. We hypothesize that shoulder and pelvic angular velocities would be highly consistent in professionals.

Methods Rotational velocities of the upper-torso and pelvis throughout the golf swing and in relation to phases of the golf swing were examined in 11 professionals and compared to 5 amateurs using three-dimensional motion analysis. Results Peak rotational velocities of professionals were highly consistent, demonstrating low variability (coefficient of variation $[\mathrm{COV}])$, particularly upper-torso rotational velocity $(\mathrm{COV}=0.086)$ and pelvic rotational velocity $(\mathrm{COV}=0.079)$ during down swing. Peak upper-torso rotational velocity and peak X-prime, the relative rotational velocity of uppertorso versus pelvis, occurred after impact in follow-through, were reduced in amateurs compared to professionals ( $p=0.005$ and $p=0.005$, respectively) and differentiated professionals from most (4/5) amateurs. In contrast, peak pelvic rotational velocity occurred in down swing. Pelvic velocity at impact was reduced in amateurs compared to professionals $(\mathrm{p}=0.019)$ and differentiated professionals from most $(4 / 5)$ amateurs.

Conclusion Golf swing rotational velocity of professionals was consistent in pattern and magnitude, offering benchmarks for amateurs. Understanding golf swing rotational biomechanics can guide swing modifications to help optimize performance and prevent injury.

Keywords Golf swing, Biomechanics, Benchmark, Angular velocity, Back pain

Department of Orthopaedic Surgery, Stanford University School of Medicine, 770 Welch Road, Suite 400, Stanford, CA, USA. Tel: +1-650-723-5308, Fax: +1-650-736-3406, E-mail: jessica.rose@stanford.edu

ORCID: Katherine M. Steele (http://orcid.org/0000-0002-4128-9387); Eugene Y. Roh (http://orcid.org/0000-0002-8139-0690); Gordhan Mahtani (http:// orcid.org/0000-0001-7549-6504); David W. Meister (http://orcid.org/0000-0002-3388-8347); Amy L. Ladd (http://orcid.org/0000-0003-0948-1034); Jessica Rose (http://orcid.org/0000-0003-0094-5525).

(c) This is an open-access article distributed under the terms of the Creative Commons Attribution Non-Commercial License (http://creativecommons.org/ licenses/by-nc/4.0) which permits unrestricted noncommercial use, distribution, and reproduction in any medium, provided the original work is properly cited. Copyright $\odot 2018$ by Korean Academy of Rehabilitation Medicine 


\section{INTRODUCTION}

Rotational biomechanics of the golf swing have been identified as important determinants of power generation, driving distance, and injury prevention [1-3]. In particular, the orientation of the pelvis and upper torso has been used as a measure of performance that can guide injury prevention [4-10]. The $\mathrm{X}$-factor, defined as the relative angular position of the pelvis and upper torso, has been shown to influence power generation and to be more highly consistent in professionals compared to amateur golf swings. Benchmark curves of angle of rotation of the pelvis, upper torso, and X-factor were reported in a cohort of 10 professional golfers throughout the golf swing and in relation to phases of the golf swing [11]. However, recent evidence has suggested that rotational velocities of the golf swing are also critical to performance and therefore can provide a basis for strategic training and injury prevention $[9,12]$. Since power is defined as the product of velocity and force, or angular velocity $\times$ moment, evaluating golf swing rotational velocities can provide insights into power generation, performance, and proper swing biomechanics. We hypothesized that shoulder and pelvis angular velocities would be highly consistent in professionals. Further, we sought to evaluate different velocities at impact or peak magnitude between professional and amateur golfers.

Golf is a popular sport with approximately 30 million people who play at least once per year in the United States, $68 \%$ of who are $30-60$ years old and $37 \%$ who are over 60 years old $[10,13]$. Around the world, over 55 million people play golf. Although golf sustains lower injury risk compared to contact sports, a successful swing is difficult to achieve and poor swing dynamics can lead to injury [13-15]. Low back pain is one of the most common injuries associated with golf. It represents $26 \%-52 \%$ of all symptoms $[6,14,16,17]$.

Mechanisms of injury tend to arise from either overuse, primarily in professionals, or trauma and improper swing biomechanics, primarily in amateurs [17-22]. Prior studies suggest that rotational biomechanics may play an important role in both preventing and treating low back pain. Improper rotational biomechanics have been shown to increase the torque, shear, and lateral-bending forces experienced by the lumbar spine in professional golfers $[6,23,24]$. Understanding rotational biomechanics of the professional and amateur golf swing can provide a basis to improve performance and decrease injury for the growing population of golfers around the world.

Several prior studies have investigated rotational biomechanics of the golf swing, including rotational velocities; however, rotational velocity throughout all phases of the golf swing has not been well studied. Zheng et al. $[9,12]$ examined angular velocity during down swing and concluded that ability to maintain separation between upper torso and pelvis segments appeared to generate higher angular velocity in professional golfers. Similarly, Myers et al. [25] reported rotational velocities of 100 recreational golfers during low, medium, and high velocity swings and found ball velocity was significantly correlated with both upper torso rotational velocity and the relative velocity of the upper torso and pelvis (X-prime). Among recreational golfers, peak upper torso and pelvis rotational velocities exceeded 700 and $400 \mathrm{deg} / \mathrm{s}$, respectively, demonstrating the extreme rotational dynamics of the golf swing. Horan and Kavanagh [26] evaluated forward tilt, lateral tilt, and axial rotational velocity of the head, trunk, and pelvis during the down swing portion of the golf swing of professional golfers. They demonstrated that the trunk had the highest peak velocities and found a high correlation between trunk and pelvic velocity. These prior studies have evaluated rotational velocities at specific points or periods during golf swing, which provide key insights into parts of the golf swing but cannot be used for benchmark comparisons to evaluate pattern of rotational velocities over the entire swing. This research is one of the first studies to assess consistency of rotational velocity over the entire golf swing.

The purpose of this study was to examine rotational velocity throughout the golf swing and in relation to phases of the golf swing among professional golfers, and to compare these benchmark curves to a sample of amateurs. Specifically, we measured the magnitude and timing of upper torso, pelvis, and X-prime angular velocities and hypothesized that these angular velocities would be highly consistent in professionals and differ at impact and in peak magnitude between professional and amateur golfers.

\section{MATERIALS AND METHODS}

\section{Motion analysis protocol}

All testing was performed indoors at the Motion \& 
Gait Analysis Laboratory at Lucille Packard Children's Hospital and the protocol was approved by the Stanford University Institutional Review Board (Protocol 11910). Three-dimensional motion analysis was used to evaluate the golf swing of 11 professional and 5 amateur golfers. The professional and amateur golfers were of similar age, height, and mass (Table 1). Inclusion criteria for professional golfers were male members of the PGA with no musculoskeletal injury that might impact their golf swing. The amateur golfers were male recreational golfers with no musculoskeletal injury that might impact their golf swing. To include a range of skill levels for comparison, our amateur golfers included one with a low handicap (handicap $=4$ ), one with a medium handicap (handicap=15), one with a high handicap (handicap=30), and two novices who did not play regularly (handicap unknown). All participants provided informed consent.

For each subject, a simplified marker set using reflective markers with diameter of $1-\mathrm{cm}$ was used to evaluate rotational velocities of the pelvis and upper torso. To evaluate pelvic motion, markers were placed on the left and right anterior superior iliac spines (ASIS). To evaluate upper torso or shoulder motion, markers were placed on the left and right acromion (ACR). A marker was also placed at the distal end of the shaft at $5-\mathrm{cm}$ from the center of the club face and a plastic practice ball was wrapped in reflective tape to identify ball contact. An 8-camera motion capture system (Motion Analysis Corporation, Santa Rosa, CA, USA) was used to capture marker positions with a sampling rate of $240 \mathrm{~Hz}$.

All golfers were given an opportunity to warm up and get comfortable in the testing area before swings were recorded. The professional golfers then performed swings at three self-selected speeds that were defined as easy, medium, and hard using their own five iron. Trials were not randomized and the golfers were given verbal cues (i.e., easy, medium, hard) before each swing. The amateur golfers had less experience controlling the speed of their golf swings and thus, only performed two hard

Table 1. Participants' characteristics

\begin{tabular}{lcc}
\hline & Professional $(\mathbf{n}=\mathbf{1 1})$ & Amateur $(\mathbf{n}=\mathbf{5})$ \\
\hline Height $(\mathrm{m})$ & $1.83 \pm 0.07$ & $1.78 \pm 0.03$ \\
Mass $(\mathrm{kg})$ & $85.9 \pm 11.5$ & $77.3 \pm 8.9$ \\
Age $(\mathrm{yr})$ & $31.0 \pm 5.9$ & $28.4 \pm 6.9$ \\
\hline
\end{tabular}

Values are presented as average \pm standard deviation. swings using their own five iron.

\section{Golf cycle and parameters}

Each golf swing was analyzed based upon the phases of the golf cycle (Fig. 1). The start of back swing was defined as a vertical velocity of the club head greater than 0.2 $\mathrm{m} / \mathrm{s}$. Impact was defined as the time point immediately preceding the initial increase in ball velocity. The end of follow-through was defined as the first local minima in vertical club head displacement following impact.

The rotation of the upper torso and pelvis were calculated as the rotation of the ACR and ASIS markers with respect to the frontal plane. Subjects were positioned in the laboratory so that their starting position had the upper torso and pelvis aligned with the global frontal plane. The X-factor was calculated as the difference between the pelvic and upper torso rotation. Obliquity of the upper torso (S-factor) and pelvis (O-factor) were calculated as the angle between the horizontal plane and a line connecting the ACR for the upper torso and the ASIS for the pelvis. Rotational velocities of the upper torso, pelvis, and $\mathrm{X}$-factor (termed $\mathrm{X}$-prime) were calculated as rates of change of the upper torso, pelvis, and $\mathrm{X}$-factor angles over the golf cycle. Upper torso and pelvic angles of rotation were previously reported in an over-lapping cohort [11].

Club head velocity was calculated from the change in position of the marker attached to the distal end of the shaft between impact and the next time point. Since the marker on the distal end of the shaft was located $5 \mathrm{~cm}$ from the center of the club face, we approximated club head velocity by fitting a circle to the trajectory of the distal shaft in the 50 time points immediately preceding and

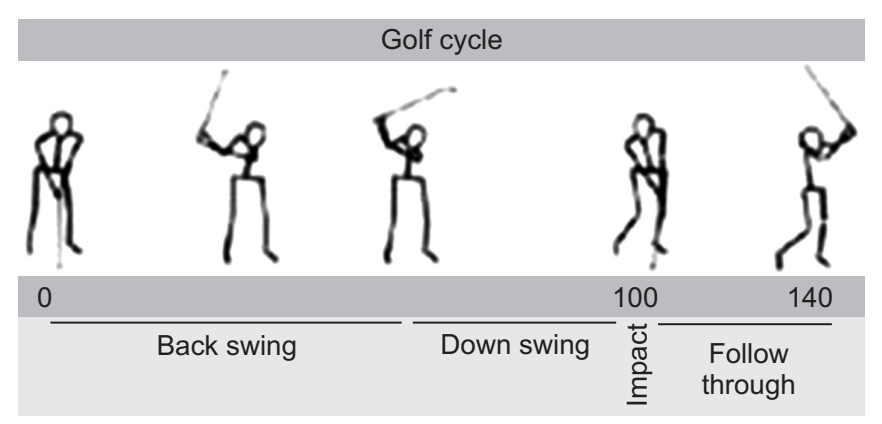

Fig. 1. The golf swing of each subject was normalized to the golf swing cycle, with values ranging from 0-140, with impact at 100 . 
after impact and adding $5 \mathrm{~cm}$ to the radius of the resulting circle.

The average rotation and rotational velocities of the upper torso, pelvis, and X-factor were compared between the professionals and amateurs using non-parametric Mann-Whitney tests with significance level set at $\mathrm{p}<0.05$. We also examined correlations $\left(\mathrm{R}^{2}\right)$ between each param- eter and club head velocity for professionals.

\section{RESULTS}

The basic marker system used in this study was able to characterize the position, velocity, and global orientation of the upper torso and pelvis in professional and
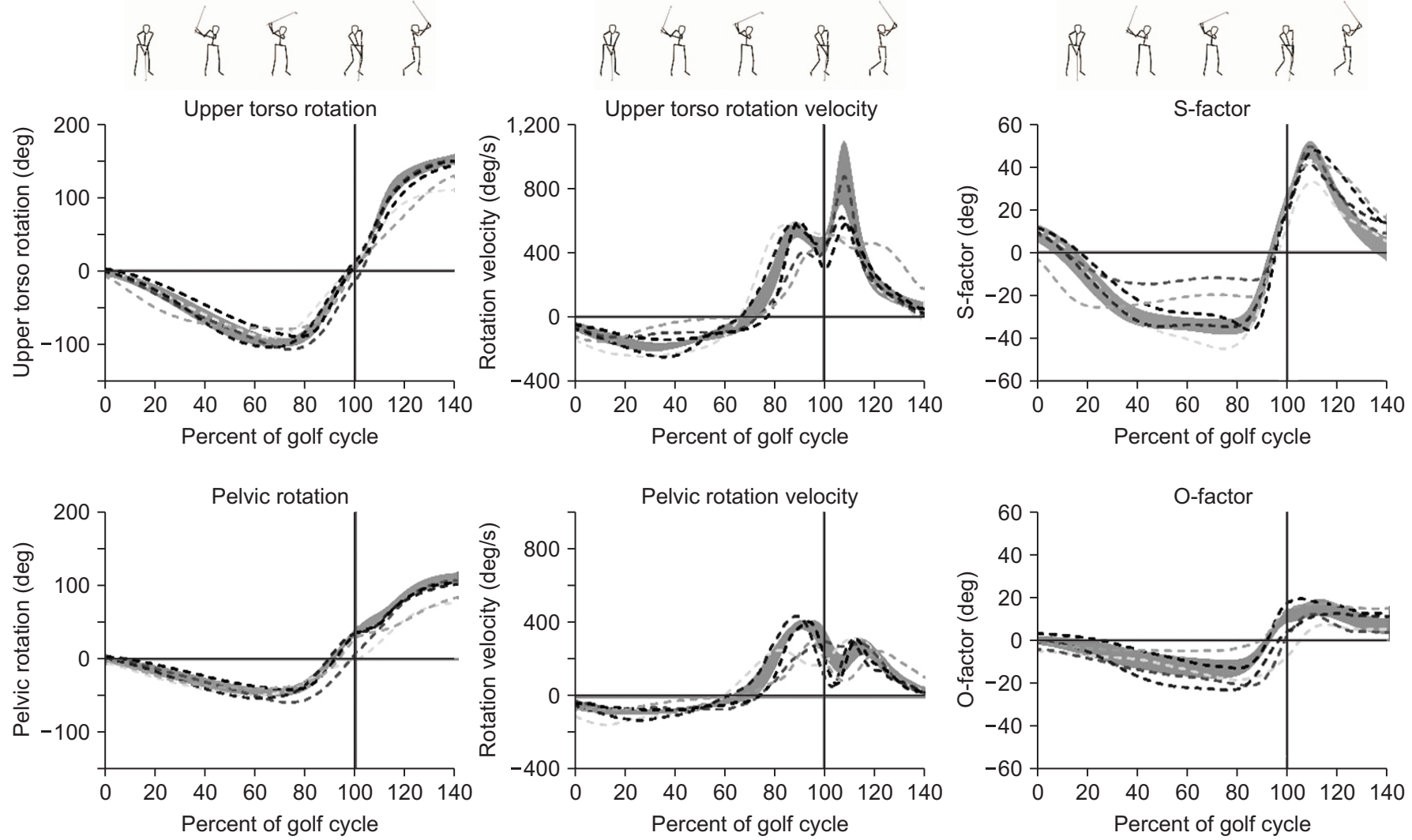

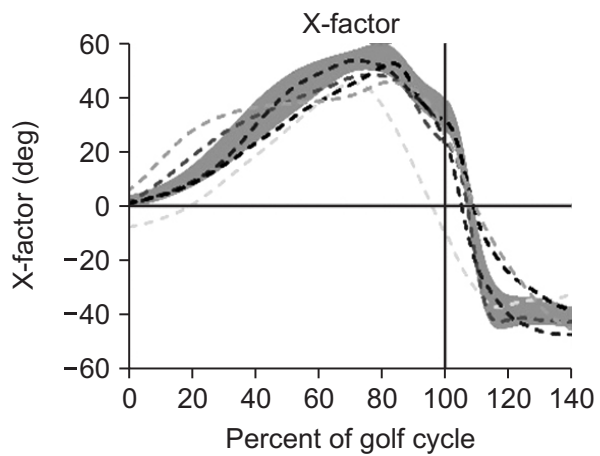

Professionals: Amateurs:

Hard swing -..-... Handicap 4

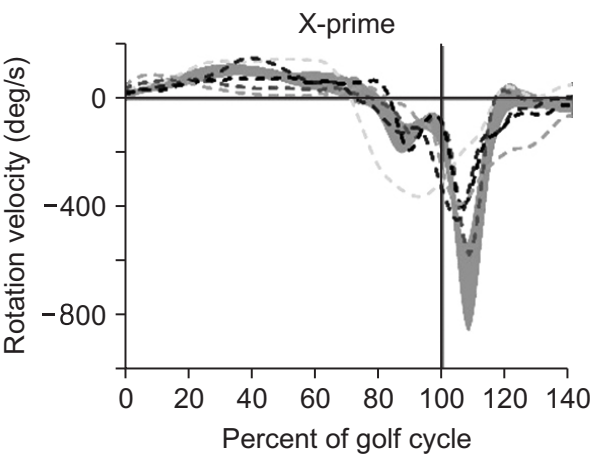

Handicap 15

Handicap 30

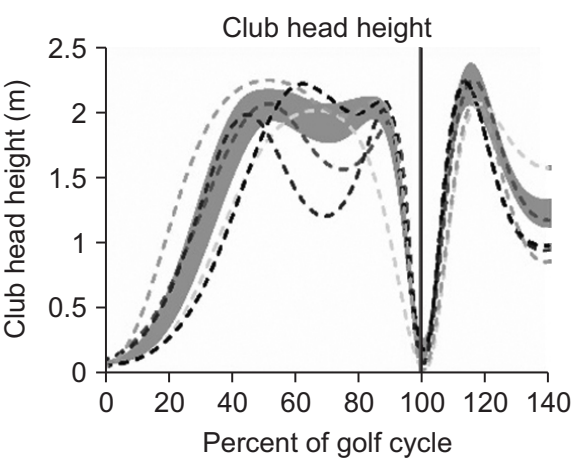

Handicap NA

Handicap NA

Fig. 2. The position (left), velocity (center), and global orientation (right) of the professional and amateur golfers. The gray area shows average \pm one standard deviation of the hard swing of the professionals. The dashed lines show average kinematics of each amateur golfer at different handicaps. 
amateur golfers (Fig. 2). The kinematics of the hard swing of professionals was consistent between individuals and provided a baseline for comparison with amateur golfers. Upper torso position, pelvic position, and X-factor demonstrated similar patterns in professionals and amateurs when normalized to the golf cycle (left column in Fig. 2). During back swing, the upper torso rotation preceded the pelvis with a greater total rotation, followed by a rapid rotation of the pelvis and upper torso during down swing. The X-factor, or relative rotation between the upper torso and pelvis, was positive throughout back swing, down swing, and at impact. The S-factor, or tilt of shoulders, the $\mathrm{O}$-factor, or tilt of the pelvis, and club head height during golf swing are also graphed, as previously reported [11].

Rotational velocities of professionals were relatively consistent between individuals and provided a baseline for comparison with amateur golfers (center column in Fig. 2). The coefficient of variation (COV) of rotational velocity of professional swings was relatively low, particularly for upper torso and pelvic rotational velocity during down swing (Table 2). Upper torso and pelvic rotational velocities and X-prime exhibited peaks before and after impact.

Peak upper torso rotational velocity occurred in followthrough was significantly reduced in amateurs compared to professionals $(\mathrm{p}=0.005)$ (top row in Figs. 2, 3) and differentiated professionals from most (4/5) amateurs. Upper torso rotational velocity during down swing or at impact was not significantly different between amateurs and professionals. In contrast, peak pelvic rotational velocity occurred in down swing. Average pelvic rotational velocity at impact was significantly lower in amateurs compared to that in professionals $(\mathrm{p}=0.019)$ (center row in Figs. 2, 3) and differentiated professionals from most (4/5) amateurs. More experienced amateurs (handicap of 4 and 15) had similar pelvic rotational velocity as professionals during down swing, but had reduced pelvic rota- tional velocity at impact.

In professionals, X-prime, or the relative rotational velocity of the upper torso and pelvis, remained near zero during back swing and increased during down swing. Xprime demonstrated a pattern of two peaks, one peak occurred in down swing just prior to impact and a second larger peak during follow-through, just after impact. In amateurs, X-prime was variable during down swing and at impact; peak X-prime occurred during followthrough, was significantly lower compared to professionals ( $\mathrm{p}=0.005)$ (bottom row in Figs. 2, 3) and differentiated professionals from most (4/5) amateurs.

Rotational velocities of professional's easy, medium, and hard golf swings are shown in Fig. 4, along with average values for amateur hard swings. Amateurs exhibited rotational velocities that were similar to the easy golf swing for professionals. Professionals increased both upper torso and pelvic rotational velocities during down swing, impact, and follow-through as they increased swing speed from easy to medium to hard. Rotational velocity of the upper torso, pelvis, and X-prime were significantly correlated with club head velocity at impact with average correlation coefficient $\left(\mathrm{R}^{2}\right)$ of $0.48,0.48$, and 0.44 , respectively $(\mathrm{p}<0.05)$.

\section{DISCUSSION}

We analyzed angular velocity of the upper torso and pelvis throughout the golf swing and in relation to phases of the golf swing, and compared these benchmarks between professional and amateur golfers. We found that professionals demonstrated consistent patterns of upper torso and pelvic rotational velocities throughout the golf swing. Rotational velocities demonstrated a unique pattern with two peaks occurring before and after impact, with a slight reduction in rotational velocity at impact. Upper torso angular velocity demonstrated a greater

Table 2. Peak rotational velocities (deg/s) of the professional golf swing

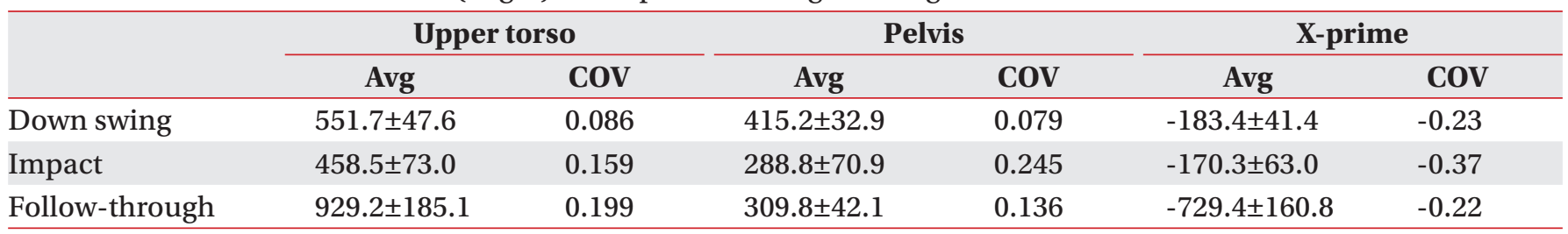

Values are presented as average \pm standard deviation.

$\mathrm{COV}$, coefficient of variation. 


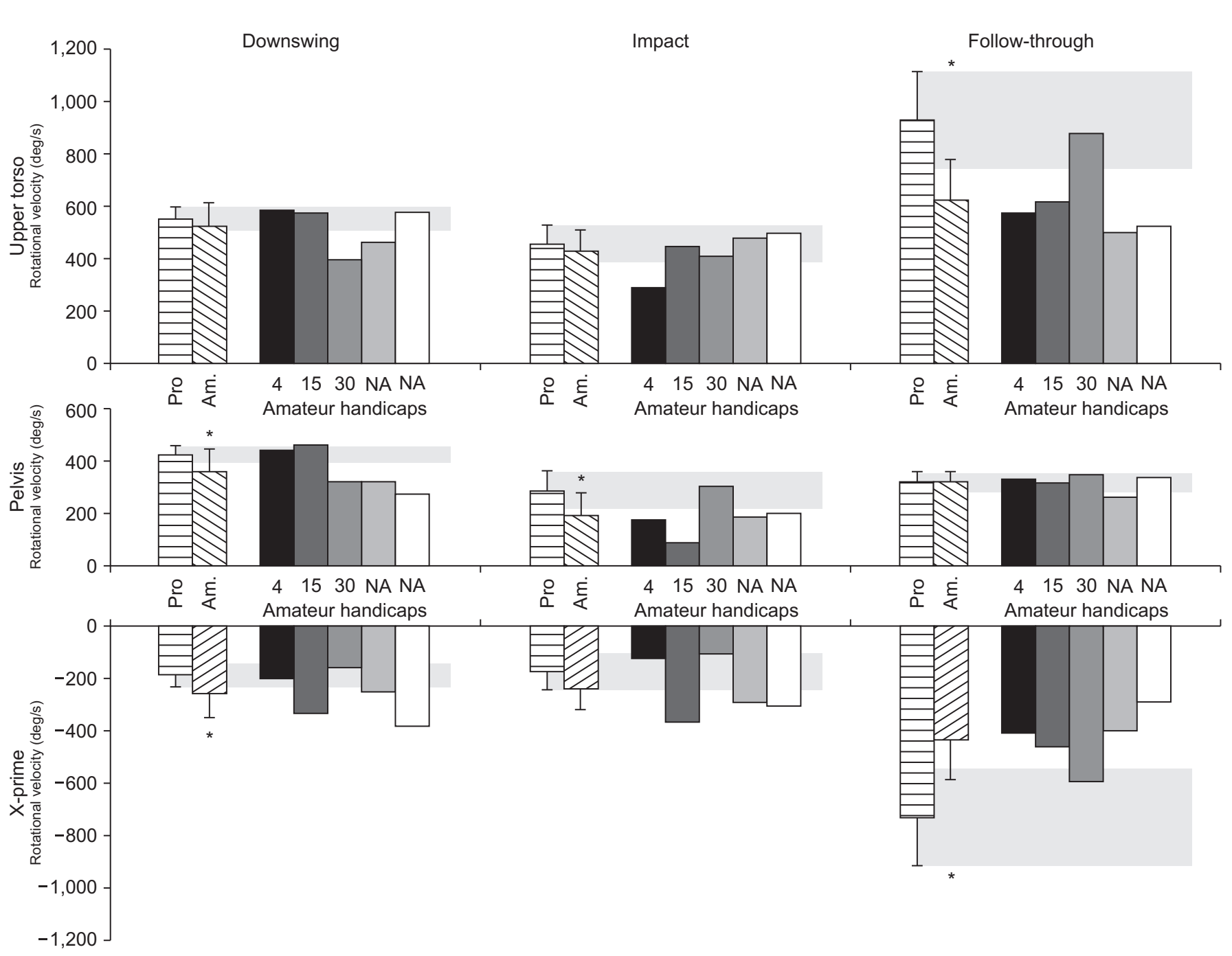

Fig. 3. Comparison of upper torso rotational velocity (top), pelvic rotational velocity (center), and X-prime rotational velocity (bottom) of the professionals and amateurs. The peak value during down swing, the value at impact, and the peak value in follow-through of each variable is shown. Since skill level varied between amateurs, the individual values of each amateur are shown for each variable. The average \pm one standard deviation of professionals' hard swings is shown in the horizontal striped bar and gray shaded area. * indicates significant difference between the average value of professional hard swing (gray) and amateurs (diagonal striped bar).
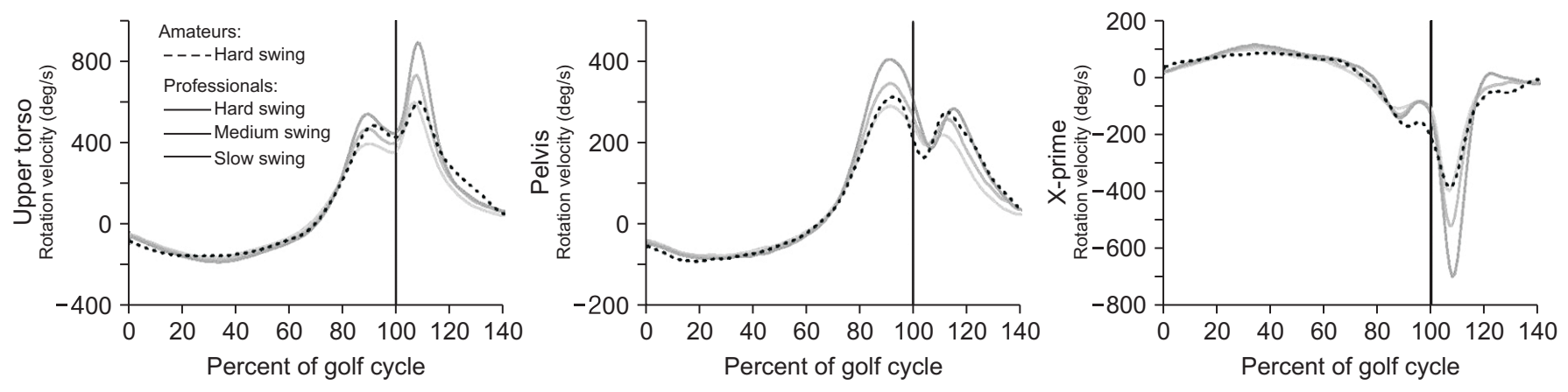

Fig. 4. Change in upper torso rotational velocity (top), pelvic rotational velocity (center), and X-prime rotational velocity (bottom) with swing speed. The amateur's velocity upper torso rotational (dashed) was similar to the slow swing of the professionals (light gray), rather than the medium swing (medium gray) or hard swing (dark gray). 
range than pelvic rotational velocity during the golf swing in both professionals and amateurs. In professionals, peak pelvic velocity occurred just prior to impact and peak upper torso velocity and X-prime occurred just after impact. Peak upper torso rotational velocity occurred in follow-through, was reduced in amateurs compared to professionals, and differentiated professionals from most amateurs. Peak pelvic rotational velocity occurred during down swing. Pelvic rotational velocity at impact was significantly lower in amateurs compared to professionals, and differentiated professionals from most amateurs. Peak X-prime was significantly reduced in amateurs compared to the professionals, and differentiated professionals from most (4/5) amateurs. The ability to record relevant upper torso and pelvic rotational velocities using this basic model has positive implications for real-time assessment of rotational velocity of golf swing using inertial sensors outside a laboratory setting.

While professionals maintained upper torso angular velocity during follow-through, in amateurs, upper torso angular velocity dropped rapidly after impact (Fig. 2). Amateurs appeared to stop their swing abruptly after impact, whereas professionals continued to finish swings, indicating that professionals had more continuous and fluid rotational mechanisms. Prior studies that examined only the down swing, reported that maximum club head velocity and peak upper torso rotational velocities occurred near the end of down swing before impact $[9,26]$. In contrast, the current study found that peak upper torso rotational velocity occurred just after impact and was substantially higher (e.g., $929 \mathrm{deg} / \mathrm{s}$ ) compared to the study (491 deg/s) of Horan and Kavanagh [26]. These findings suggest that modulation of upper torso rotational velocity during follow-through may have important implications for performance training and injury prevention.

Pelvic rotational velocity at impact was significantly lower in amateurs compared to professionals. Low handicap golfers had pelvic rotational velocities similar to professionals, while the golfer with handicap of 30 and two novices had slower pelvic velocities. In follow-through pelvic velocity was not significantly different between pros and amateurs. Zheng et al. [9] reported that peak pelvic rotational velocity occurred at impact. In contrast, the current study found that peak pelvic rotational velocity occurred just prior to impact. The peak pelvic ro- tational velocities during down swing in this study were similar to the previous reported results of professionals from Horan and Kavanagh [26] (415 deg/s vs. 464 deg/s).

The current study is the first to describe the kinematic factor of 'X-prime' defined by a relative rotational velocity of the upper torso and pelvis, throughout the golf swing. The results demonstrated that X-prime remained close to zero during the early phases of the golf swing and demonstrated a rapid increase before impact. The magnitude of X-prime at impact in the professional golfers was slightly reduced compared to results reported by Myers et al. [25] in recreational golfers (e.g., $170 \mathrm{deg} / \mathrm{s}$ vs. $272 \mathrm{deg} / \mathrm{s}$ at impact). While X-prime did not differentiate the highest performing amateur from professionals during all phases of the golf swing, there was a significant and distinguishing difference in follow-through (Figs. $2,3)$. The difference in X-prime in follow-through arose primarily from differences in upper torso angular velocity during follow-through and suggests that maintaining faster upper torso angular velocity during follow-through may be a distinguishing feature of the professional golf swing.

Peak upper torso rotational velocity occurred just after impact $(>1,000 \mathrm{deg} / \mathrm{s}$ in pros and $600 \mathrm{deg} / \mathrm{s}$ in low handicap amateur golfers) (Fig. 2). Amateurs' hard swing showed similar upper torso velocity as Professionals' slow swing. These findings suggest that a rapid upper torso rotational velocity and X-prime during followthrough may be important elements of optimal swing mechanics. In the current study, upper torso and pelvic rotational velocity and X-prime demonstrated a linear increase from slow to medium to hard swings. Meister et al. [11] previously reported a linear increase in peak upper torso rotation angle from slow to medium to hard swings, but did not report on rotational velocity, in an over lapping cohort. These findings suggest that upper torso rotation during follow-through phase of the golf swing likely contributes to power generation and to a smooth swing pattern that may prevent injury, and thus may warrant specific training strategies.

The eleven professional golfers examined in the current study demonstrated consistent rotational velocity swing patterns that allowed for the generation of normal benchmark curves, based on hard swing kinematics, normalized to the golf swing cycle. Benchmark curves generated from the consistent golf swing of professional golfers 
can guide swing modifications for individualized training to provide milestones for performance while preventing injury.

Previous studies have reported that poor golf swing mechanics are one of the leading causes of golf-related injuries, especially for amateur players [21,22]. Low back injuries are one of the most prevalent injuries in golf $[21,27]$ and have been shown to be related to an excessive $\mathrm{X}$-factor $[23,28]$. Benchmark curves can provide important guidelines and feedback that can be used to prevent excessive rotation and risk of injury, while optimizing performance. We found that while rotational velocity was lower in our sample of amateurs compared to pros, upper torso rotational velocity exhibited an abrupt decrease after impact compared to pros. This suggests that modulation of upper torso rotational velocity during the entire golf swing may be an important factor to consider to achieve a smooth swing pattern, both for performance as well as injury prevention in amateur golfers. A precise understanding of the optimal rotational biomechanics during the golf swing may guide swing modifications to help prevent or aid in the treatment of injury $[29,30]$.

The current study was limited in that data were necessarily collected in an indoor environment where the true outcomes of shots were unknown. Use of a plastic ball might have affected conditions at impact. The study was designed to determine factors that may contribute to power generation and injury prevention, as indicated by rotational velocities of professional in relation to club velocity at impact, a commonly used measure of power generation, but not actual driving distance $[5,8]$. Additionally, only male golfers were included in this study, and similar benchmark curves for female professional golfers are needed and would provide insight into differences in rotational biomechanics. An ideal men's golf swing should not be generalized for female golfers $[3,12]$. We tested golfers using a 5-iron club and prior studies have reported that peak and impact club head velocities vary with different types of clubs, and may influence rotational biomechanics [7].

In conclusion, data from this study supports our hypothesis that golf swing rotational velocity of the upper torso and pelvis are consistent in professionals. Further, we found that there were common differences between professionals and amateurs with a range of skill levels that may be useful for coaching. Golfers with lower hand- icaps demonstrated rotational velocities closer to professionals. Benchmark curves derived from consistent angular velocities of pros can be used as guidelines for golf swing injury prevention and performance training. While rotational velocity was lower in amateurs compared to pros, upper torso rotational velocity exhibited an abrupt decrease after impact, in contrast to pros. This suggests that modulation of upper torso rotational velocity may be an important factor in achieving a smooth swing pattern for both injury prevention and performance in amateur golfers. An advantage of this methodology is that only a limited number of markers $(n=5)$ were required for motion analysis and similar methods could be applied using inertial sensors or other wearable technology. Further study is required to develop training strategies that can effectively improve patterns of upper torso and pelvic rotational velocity and X-prime in amateur golfers.

\section{CONFLICT OF INTEREST}

No potential conflict of interest relevant to this article was reported.

\section{ACKNOWLEDGMENTS}

This study was supported in part by the Medical Scholars Research Program at Stanford University School of Medicine, the National Science Foundation Graduate Research Fellowship under grant number DGE-1147470, and Media-X, Stanford University.

\section{REFERENCES}

1. Evans K, Refshauge KM, Adams R. Measurement of active rotation in standing: reliability of a simple test protocol. Percept Mot Skills 2006;103:619-28.

2. Evans K, Refshauge KM, Adams RD, Barrett R. Swing kinematics in skilled male golfers following putting practice. J Orthop Sports Phys Ther 2008;38:425-33.

3. Horan SA, Evans K, Morris NR, Kavanagh JJ. Thorax and pelvis kinematics during the downswing of male and female skilled golfers. J Biomech 2010;43:1456-62.

4. Ball KA, Best RJ. Different centre of pressure patterns within the golf stroke I: cluster analysis. J Sports Sci 2007;25:757-70.

5. Ball KA, Best RJ. Different centre of pressure patterns 
within the golf stroke II: group-based analysis. J Sports Sci 2007;25:771-9.

6. Gluck GS, Bendo JA, Spivak JM. The lumbar spine and low back pain in golf: a literature review of swing biomechanics and injury prevention. Spine J 2008;8:77888.

7. Hume PA, Keogh J, Reid D. The role of biomechanics in maximising distance and accuracy of golf shots. Sports Med 2005;35:429-49.

8. Teu KK, Kim W, Fuss FK, Tan J. The analysis of golf swing as a kinematic chain using dual Euler angle algorithm. J Biomech 2006;39:1227-38.

9. Zheng N, Barrentine SW, Fleisig GS, Andrews JR. Kinematic analysis of swing in pro and amateur golfers. Int J Sports Med 2008;29:487-93.

10. Statistic Brain Research Institute. Golf player demographic statistics 2012 [Internet]. Los Angeles: Statistic Brain Research Institute; c2018 [cited 2018 Sep 15]. Available from: http://www.statisticbrain.com/golfplayer-demographic-statistics/.

11. Meister DW, Ladd AL, Butler EE, Zhao B, Rogers AP, Ray CJ, et al. Rotational biomechanics of the elite golf swing: benchmarks for amateurs. J Appl Biomech 2011;27:242-51.

12.Zheng N, Barrentine SW, Fleisig GS, Andrews JR. Swing kinematics for male and female pro golfers. Int J Sports Med 2008;29:965-70.

13. Farrally MR, Cochran AJ, Crews DJ, Hurdzan MJ, Price RJ, Snow JT, et al. Golf science research at the beginning of the twenty-first century. J Sports Sci 2003;21:753-65.

14. Fradkin AJ, Cameron PA, Gabbe BJ. Golf injuries: common and potentially avoidable. J Sci Med Sport 2005;8:163-70.

15. Werner D. Driving toward prevention. Phys Ther Prod 2000;5:12-5.

16. Fradkin AJ, Cameron PA, Gabbe BJ. Is there an association between self-reported warm-up behaviour and golf related injury in female golfers? J Sci Med Sport 2007;10:66-71.
17. Gosheger G, Liem D, Ludwig K, Greshake O, Winkelmann W. Injuries and overuse syndromes in golf. Am J Sports Med 2003;31:438-43.

18. Batt ME. A survey of golf injuries in amateur golfers. Br J Sports Med 1992;26:63-5.

19. Batt ME. Golfing injuries: an overview. Sports Med 1993;16:64-71.

20. Finch C, Sherman C, James T. The epidemiology of golf injuries in Victoria, Australia: evidence from sports medicine clinics and emergency department presentations. In: Farrally MR, Cochran AJ, editors. Science and Golf III: Proceedings of the 1998 World Scientific Congress of Golf. Champaign: Human Kinetics; 1999. p. 73-82.

21. McHardy A, Pollard H, Luo K. Golf injuries: a review of the literature. Sports Med 2006;36:171-87.

22. Theriault G, Lachance P. Golf injuries: an overview. Sports Med 1998;26:43-57.

23. Lindsay D, Horton J. Comparison of spine motion in elite golfers with and without low back pain. J Sports Sci 2002;20:599-605.

24. McCarroll JR, Gioe TJ. Professional Golfers and the Price They Pay. Phys Sportsmed 1982;10:64-70.

25. Myers J, Lephart S, Tsai YS, Sell T, Smoliga J, Jolly J. The role of upper torso and pelvis rotation in driving performance during the golf swing. J Sports Sci 2008;26:181-8.

26. Horan SA, Kavanagh JJ. The control of upper body segment speed and velocity during the golf swing. Sports Biomech 2012;11:165-74.

27. McHardy A, Pollard H. Muscle activity during the golf swing. Br J Sports Med 2005;39:799-804.

28. Lindsay DM, Horton JF. Trunk rotation strength and endurance in healthy normals and elite male golfers with and without low back pain. N Am J Sports Phys Ther 2006;1:80-9.

29. Parziale JR. Healthy swing: a golf rehabilitation model. Am J Phys Med Rehabil 2002;81:498-501.

30. Parziale JR, Mallon WJ. Golf injuries and rehabilitation. Phys Med Rehabil Clin N Am 2006;17:589-607. 